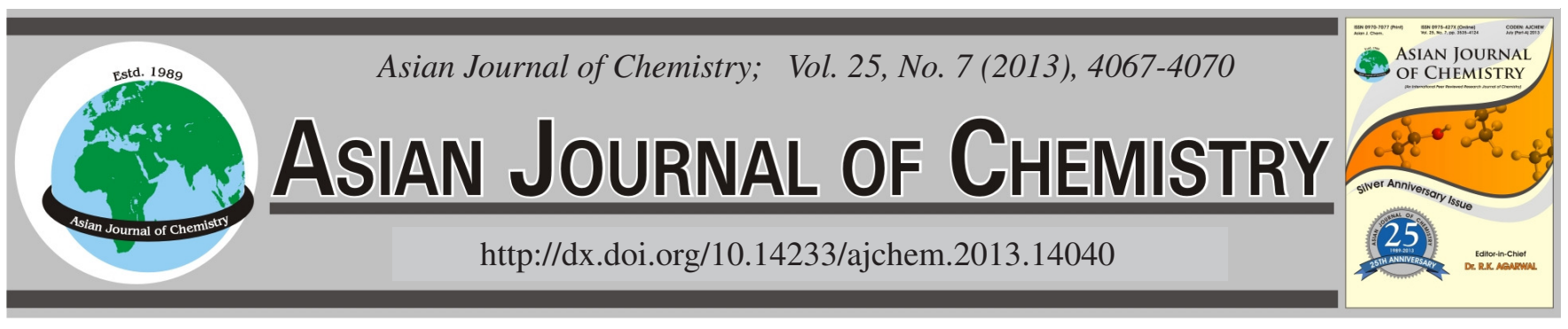

\title{
Synthesis, Structure and Biological Activities of 2,2-Dichloro-1-(4-ethoxyphenyl)- cyclopropanyl Substituted Piperidin-1-yl Ketone
}

\author{
Na-Bo Sun ${ }^{*}$, JiAn-Zhong Jin, Chao Lei and Wei Ke
}

College of Biology and Environmental Engineering, Zhejiang Shuren University, Hangzhou, 310015, P.R. China

*Corresponding author: E-mail: nabosun@gmail.com

Ten new amide-type compounds, which are pyrethroids containing piperidine, are synthesized from 1-(4-ethyoxyphenyl)-2,2dichlorocyclopropane-1-carboxylic acid and substituted piperidine. Their structures were confirmed by ${ }^{1} \mathrm{H}$ NMR, MS and elemental analysis. The crystal structure of $\mathbf{4 a}$ and $\mathbf{4 h}$ were determined by X-ray diffraction analysis. The bioassay results indicated that they showed moderate activity.

Key Words: Pyrethroid, Cycloprothrin, Piperidine, Synthesis, Structure.

\section{INTRODUCTION}

Cyclopropane derivatives, as a kind of highly bioactive compounds, have been studied broadly ${ }^{1}$. At the end of 1940s, cyclopropane compounds, such as pyrethroids, were marketed as low toxic insecticides. Since the first pyrethroids insecticideAllethrin was found, a large variety of pyrethroids derivatives have been synthesized and lots of them, such as deltamethrin, cypermethrin, bifenthrin, fenvalerate, tefluthrin etc. are commercially available ${ }^{2}$. From then on, many biologically active and structurally stable cyclopropane compounds had been synthesized $^{3}$.

In recent years, synthesis of broader spectrum and highly bioactive substituted cyclopropane compounds, especially heterocycle substituted ones, becomes the main stream in the agriculture chemistry field. Also, from the structure-activity relationships of these pyrethroids, it is showed that the ester group was replaced by other functional groups, such as amide group from numerous reports. Due to the amide group exhibited the good biological activity, such as herbicidal activity, fungicidal activity, insecticide activity, etc. ${ }^{4}$. In line with our continuous efforts to synthesize bioactive lead compounds for crop protection, the title compounds were designed by introducing amide and piperidine pharmacophore into the cyclopropane scaffold and their biologicial activity tested. Then, the single crystal of the title compound was determined. The preliminary biological test showed that the synthesized compound has moderate fungicidal activity.

\begin{abstract}
$------------1$
\end{abstract}
\section{EXPERIMENTAL}

Melting points were determined by an X-4 apparatus and uncorrected. ${ }^{1} \mathrm{H}$ NMR spectra were measured on a Bruker Avance 400 DMX instrument using TMS as an internal standard and $\mathrm{CDCl}_{3}$ as the solvent. Mass spectra were recorded on a HP 5989B mass detector instrument. Elemental analyses were performed on a Carlo erba EA1110 elemental analyzer. All the reagents are of analytical grade or freshly prepared before use.

To 1-(4-ethyoxyphenyl)-2,2-dichlorocyclopropane-1carboxylic acid $(2.75 \mathrm{~g}, 10 \mathrm{mmol}$ ) was added thionyl chloride $(20 \mathrm{~mL})$ and the mixture was refluxed for $2 \mathrm{~h}$ to give acid chloride. To a mixture of compound $3(8.8 \mathrm{mmol})$ and $\mathrm{Et}_{3} \mathrm{~N}$ (1.2 g, $12 \mathrm{mmol})$ in $\mathrm{CH}_{2} \mathrm{Cl}_{2}(20 \mathrm{~mL})$ was added 2. The mixture was stirred for 10-25 h. The corresponding amide precipitated immediately. The product was washed with $\mathrm{HCl}$, dried and was purified by chromatography on silica gel using petroleum ether $\left(60-90{ }^{\circ} \mathrm{C}\right)$ and ethyl acetate as the eluent to afford the title compounds $\mathbf{4 a - 4 j}$.

2,2-Dichloro-1-(4-ethoxyphenyl)cyclopropanyl piperidin-1-yl ketone (4a). Colourless crystal; yield, $92 \%$; m.p., $121-123{ }^{\circ} \mathrm{C} ;{ }^{1} \mathrm{H} \mathrm{NMR}\left(\mathrm{CDCl}_{3}\right) \delta: 1.41(\mathrm{t}, J=7.2 \mathrm{~Hz}, 3 \mathrm{H}$, $\left.\mathrm{CH}_{3}\right), 1.49-1.59$ (m, 6H, piperdine $\left.\mathrm{H}\right), 1.99(\mathrm{~d}, J=7.2 \mathrm{~Hz}, 1 \mathrm{H}$, cyclopropane $\mathrm{H}), 2.35$ (d, $J=7.2 \mathrm{~Hz}, 1 \mathrm{H}$, cyclopropane $\mathrm{H})$, 3.44-3.67 (m, 4H, piperdine $\mathrm{H}), 4.00-4.05\left(\mathrm{~m}, 2 \mathrm{H}, \mathrm{CH}_{3} \mathrm{CH}_{2} \mathrm{O}\right)$, 6.85-6.88 (m, 2H, Ph), 7.86-7.88 (m, 2H, Ph); Ms m/z (relative intensity/\%): 341([M-1] $\left.]^{+}, 100\right), 306$ (48), 277 (56), 222 
(30), 193 (53), 165 (29), 131 (86), 112 (30), 97 (32), 84 (41); Anal. calcd. for $\mathrm{C}_{17} \mathrm{H}_{20} \mathrm{NO}_{2} \mathrm{Cl}_{2}$ (\%): C 59.66, H 6.18, N 4.09, found: C 59.88, H 6.21, N 4.02.

2,2-Dichloro-1-(4-ethoxyphenyl)cyclopropanyl 4-methylpiperidin-1-yl ketone (4b). Yellow crystal; yield, 64.6\%; m.p., 82-84; ${ }^{1} \mathrm{H}$ NMR $\left(\mathrm{CDCl}_{3}\right)$ $\delta: 0.83\left(\mathrm{~d}, J=7.2 \mathrm{~Hz}, 3 \mathrm{H}, \mathrm{CH}_{3}\right)$, $1.40\left(\mathrm{t}, J=7.2 \mathrm{~Hz}, 3 \mathrm{H}, \mathrm{CH}_{3} \mathrm{CH}_{2} \mathrm{O}\right), 1.48-1.63(\mathrm{~m}, 5 \mathrm{H}$, piperdine H), 1.99 (d, $J=7.2 \mathrm{~Hz}, 1 \mathrm{H}$, cyclopropane H), 2.53 (d, $J=7.2$ $\mathrm{Hz}, 1 \mathrm{H}$, cyclopropane $\mathrm{H}), 3.57-3.70(\mathrm{~m}, 4 \mathrm{H}$, piperdine $\mathrm{H})$, 4.01-4.05 (m, 2H, $\left.\mathrm{CH}_{3} \mathrm{CH}_{2} \mathrm{O}\right), 6.80-6.82(\mathrm{~m}, 2 \mathrm{H}, \mathrm{Ph}), 7.85-$ 7.87 (m, 2H, Ph); Ms m/z (relative intensity/\%): 355 ([M-1] $]^{+}$, 100), 320 (63), 291 (60), 222 (76), 193 (81), 165 (29), 131 (35), 112 (44), 97 (63); Anal. calcd. for $\mathrm{C}_{18} \mathrm{H}_{23} \mathrm{NO}_{2} \mathrm{Cl}_{2}(\%)$ : C 60.68, H 6.51, N 3.93, found: C 60.59, H 6.58, N 3.90.

2,2-Dichloro-1-(4-ethoxyphenyl)cyclopropanyl 3methylpiperidin-1-yl ketone (4c). Yellow crystal; yield, $60.5 \%$; m.p., $90-92{ }^{\circ} \mathrm{C} ;{ }^{1} \mathrm{H}$ NMR $\left(\mathrm{CDCl}_{3}\right) \delta: 0.88$ (d, $J=7.2$ $\left.\mathrm{Hz}, 3 \mathrm{H}, \mathrm{CH}_{3}\right), 1.41$ (t, $J=7.2 \mathrm{~Hz}, 3 \mathrm{H}, \mathrm{CH}_{3} \mathrm{CH}_{2} \mathrm{O}$ ), $1.45-1.56$ (m, 5H, piperdine $\mathrm{H}), 1.98(\mathrm{~d}, J=7.2 \mathrm{~Hz}, 1 \mathrm{H}$, cyclopropane H), 2.48 (d, $J=7.2 \mathrm{~Hz}, 1 \mathrm{H}$, cyclopropane H), 3.51-3.69 (m, $4 \mathrm{H}$, piperdine $\mathrm{H}), 4.01-4.04\left(\mathrm{~m}, 2 \mathrm{H}, \mathrm{CH}_{3} \mathrm{CH}_{2} \mathrm{O}\right)$, 6.84-6.86 (m, 2H, Ph), 7.82-7.84 (m, 2H, Ph); Ms $\mathrm{m} / \mathrm{z}$ (relative intensity/\%): 355 ([M-1] $\left.]^{+}, 100\right), 320$ (32), 291 (66), 222 (26), 193 (36), 165 (71), 131 (35), 112 (56), 97 (30), 84 (71); Anal. calcd. for $\mathrm{C}_{18} \mathrm{H}_{23} \mathrm{NO}_{2} \mathrm{Cl}_{2}(\%)$ : $\mathrm{C} 60.68, \mathrm{H}$ 6.51, N 3.93, found: C 60.50, H 6.45, N 4.00.

2,2-Dichloro-1-(4-ethoxyphenyl)cyclopropanyl 2ethylpiperidin-1-yl ketone (4d). White crystal; yield, $93.5 \%$; m.p., $134-137{ }^{\circ} \mathrm{C} ;{ }^{1} \mathrm{H}$ NMR $\left(\mathrm{CDCl}_{3}\right) \delta: 0.85(\mathrm{t}, J=7.2 \mathrm{~Hz}, 3 \mathrm{H}$, $\left.\mathrm{CH}_{3}\right), 1.41\left(\mathrm{t}, J=7.2 \mathrm{~Hz}, 3 \mathrm{H}, \mathrm{CH}_{3} \mathrm{CH}_{2} \mathrm{O}\right), 1.50-1.63(\mathrm{~m}, 8 \mathrm{H}$, piperdine $\mathrm{H}$ and $\left.\mathrm{CH}_{2} \mathrm{CH}_{3}\right), 1.90(\mathrm{~d}, J=7.2 \mathrm{~Hz}, 1 \mathrm{H}$, cyclopropane $\mathrm{H}), 2.41$ (d, $J=7.2 \mathrm{~Hz}, 1 \mathrm{H}$, cyclopropane $\mathrm{H}), 3.19-3.25$ (m, 3H, piperdine $\mathrm{H}$ ), 4.00-4.05 (m, $2 \mathrm{H}, \mathrm{CH}_{2} \mathrm{O}$ ), 6.85-6.87 $(\mathrm{m}, 2 \mathrm{H}, \mathrm{Ph}), 7.49-7.51(\mathrm{~m}, 2 \mathrm{H}, \mathrm{Ph}) ; \mathrm{Ms} \mathrm{m} / \mathrm{z}$ (relative intensity/\%): 369 ([M-1] $\left.]^{+}, 95\right), 340$ (44), 220 (100), 195 (33), 162 (55), 140 (83), 131 (64), 84 (47); Anal. calcd. for $\mathrm{C}_{19} \mathrm{H}_{25} \mathrm{NO}_{2} \mathrm{Cl}_{2}$ (\%): C 61.62, H 6.80, N 3.78, found: C 61.81, H 6.88, N 3.72.

2,2-Dichloro-1-(4-ethoxyphenyl)cyclopropanyl 4ethylpiperidin-1-yl ketone (4e). White crystal; yield, $86.4 \%$; $\mathrm{mp}, 125-128{ }^{\circ} \mathrm{C} ;{ }^{1} \mathrm{H} \mathrm{NMR}\left(\mathrm{CDCl}_{3}\right) \delta: 0.88(\mathrm{t}, J=7.2 \mathrm{~Hz}, 3 \mathrm{H}$, $\left.\mathrm{CH}_{3}\right), 1.41\left(\mathrm{t}, J=7.2 \mathrm{~Hz}, 3 \mathrm{H}, \mathrm{CH}_{3} \mathrm{CH}_{2} \mathrm{O}\right), 1.2-1.62(\mathrm{~m}, 7 \mathrm{H}$, piperdine $\mathrm{H}$ and $\left.\mathrm{CH}_{2} \mathrm{CH}_{3}\right), 1.90(\mathrm{~d}, J=7.2 \mathrm{~Hz}, 1 \mathrm{H}$, cyclopropane $\mathrm{H}), 2.41$ (d, $J=7.2 \mathrm{~Hz}, 1 \mathrm{H}$, cyclopropane $\mathrm{H}), 3.19-3.25$ (m, $4 \mathrm{H}$, piperdine $\mathrm{H})$, 4.01-4.05 (m, $\left.2 \mathrm{H}, \mathrm{CH}_{3} \mathrm{CH}_{2} \mathrm{O}\right)$, 6.84$6.86(\mathrm{~m}, 2 \mathrm{H}, \mathrm{Ph}), 7.45-7.47(\mathrm{~m}, 2 \mathrm{H}, \mathrm{Ph}) ; \mathrm{Ms} \mathrm{m} / \mathrm{z}$ (relative intensity/\%): 369 ([M-1] ] , 93), 340 (30), 295 (34), 220 (100), 162 (69), 140 (68), 131 (87), 102 (28); Anal. calcd. for $\mathrm{C}_{19} \mathrm{H}_{25} \mathrm{NO}_{2} \mathrm{Cl}_{2}(\%)$ : C 61.62, H 6.80, N 3.78, found: C 61.75, H 6.86, N 3.73 .

2,2-Dichloro-1-(4-ethoxyphenyl)cyclopropanyl 2,6dimethylpiperidin-1-yl ketone (4f). Colourless crystal; yield, $56.2 \%$; m.p., $124-127{ }^{\circ} \mathrm{C} ;{ }^{1} \mathrm{H}$ NMR $\left(\mathrm{CDCl}_{3}\right) \delta: 1.11(\mathrm{~d}, J=$ $\left.7.2 \mathrm{~Hz}, 6 \mathrm{H}, \mathrm{CH}_{3}\right), 1.41\left(\mathrm{t}, J=6.8 \mathrm{~Hz}, 3 \mathrm{H}, \mathrm{CH}_{3} \mathrm{CH}_{2} \mathrm{O}\right), 1.58$ $1.78(\mathrm{~m}, 6 \mathrm{H}$, piperidine $\mathrm{H}), 1.88(\mathrm{~d}, J=7.2 \mathrm{~Hz}, 1 \mathrm{H}$, cyclopropane $\mathrm{H}), 2.23-2.28(\mathrm{~m}, 2 \mathrm{H}$, piperidine $\mathrm{H}), 3.99-4.03(\mathrm{~m}, 2 \mathrm{H}$, $\mathrm{CH}_{3} \mathrm{CH}_{2} \mathrm{O}$ ), 6.84-6.88 (m, 2H, Ph), 7.53-7.55 (m, 2H, Ph); Ms $\mathrm{m} / \mathrm{z}$ (relative intensity/\%): $369\left([\mathrm{M}-1]^{+}, 100\right), 258(15), 234$ (57), 222 (28), 195 (32), 165 (40), 140 (74), 131 (53), 112
(63), 97 (35); Anal. calcd. for $\mathrm{C}_{19} \mathrm{H}_{25} \mathrm{NO}_{2} \mathrm{Cl}_{2}$ (\%): C 61.62, $\mathrm{H}$ 6.80, N 3.78; found: C 61.69, H 6.84, N 3.75.

2,2-Dichloro-1-(4-ethoxyphenyl)cyclopropanyl 3,3dimethylpiperidin-1-yl ketone $\mathbf{( 4 g )}$. White crystal; yield,

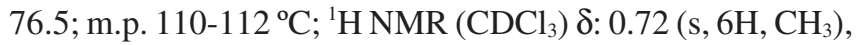
$1.41\left(\mathrm{t}, J=6.8 \mathrm{~Hz}, 3 \mathrm{H}, \mathrm{CH}_{3} \mathrm{CH}_{2}\right), 1.44-1.50$ (m, 4H, piperidine H), 1.97 (d, $J=6.8 \mathrm{~Hz}, 1 \mathrm{H}$, cyclopropane $\mathrm{H}$ ), 2.40 (d, $J$ $=7.2 \mathrm{~Hz}, 1 \mathrm{H}$, cyclopropane $\mathrm{H}), 3.10(\mathrm{t}, J=8.4 \mathrm{~Hz}, 2 \mathrm{H}$, piperidine $\mathrm{H}), 3.66(\mathrm{~s}, 2 \mathrm{H}$, piperidine $\mathrm{H}), 4.00-4.05(\mathrm{~m}, 2 \mathrm{H}$, $\mathrm{CH}_{3} \mathrm{CH}_{2} \mathrm{O}$ ), 6.85-6.87 (m, 2H, Ph), 7.49-7.51 (m, 2H, Ph); $\mathrm{Ms}$ $\mathrm{m} / \mathrm{z}$ (relative intensity/\%): $369\left([\mathrm{M}-1]^{+}, 100\right), 334$ (30), 306 (21), 270 (26), 222 (30), 193 (17), 165 (29), 140 (63), 131 (38), 112 (35), 97 (14), 84 (33); Anal. calcd. for $\mathrm{C}_{19} \mathrm{H}_{25} \mathrm{NO}_{2} \mathrm{Cl}_{2}$ (\%): C 61.62, H 6.80, N 3.78, found: C 61.58, H 6.83, N 3.77.

2,2-Dichloro-1-(4-ethoxyphenyl)cyclopropanyl 2,3dimethylpiperidin-1-yl ketone (4h). Colourless crystal; yield, $90.0 \%$; m.p., 159-161 ${ }^{\circ} \mathrm{C} ;{ }^{1} \mathrm{H}$ NMR $\left(\mathrm{CDCl}_{3}\right)$ $\delta: 0.85$ (d, $J=$ $\left.6.8 \mathrm{~Hz}, 6 \mathrm{H}, \mathrm{CH}_{3}\right), 1.41\left(\mathrm{t}, J=6.8 \mathrm{~Hz}, 3 \mathrm{H}, \mathrm{CH}_{3} \mathrm{CH}_{2} \mathrm{O}\right), 1.58-$ 1.69 (m, 5H, piperidine $\mathrm{H}), 2.01(\mathrm{~d}, J=6.8 \mathrm{~Hz}, 1 \mathrm{H}$, cyclopropane $\mathrm{H}), 2.32(\mathrm{~d}, J=6.8 \mathrm{~Hz}, 1 \mathrm{H}$, cyclopropane $\mathrm{H}$ ), 2.60-2.81 (m, 3H, piperidine $\mathrm{H})$, 3.98-4.04 (m, $\left.2 \mathrm{H}, \mathrm{CH}_{3} \mathrm{CH}_{2} \mathrm{O}\right)$, 6.84$6.86(\mathrm{~m}, 2 \mathrm{H}, \mathrm{Ph}), 7.45-7.50(\mathrm{~m}, 2 \mathrm{H}, \mathrm{Ph}) ; \mathrm{Ms} m / z$ (relative intensity/\%): 369 ([M-1] $\left.]^{+}, 100\right), 334$ (19), 306 (17), 270 (8), 222 (30), 195 (22), 165 (37), 140 (68), 131 (44), 112 (47), 98 (20), 84 (34); Anal. calcd. for $\mathrm{C}_{19} \mathrm{H}_{25} \mathrm{NO}_{2} \mathrm{Cl}_{2}$ (\%): C 61.62, $\mathrm{H}$ 6.80, N 3.78; found: C 61.55, H 6.84, N 3.74.

2,2-Dichloro-1-(4-ethoxyphenyl)cyclopropanyl 3,5dimethylpiperidin-1-yl ketone (4i). White crystal; yield, 64.8; m.p., $126-129{ }^{\circ} \mathrm{C} ;{ }^{1} \mathrm{H}$ NMR $\left(\mathrm{CDCl}_{3}\right) \delta: 0.86(\mathrm{~d}, J=7.2 \mathrm{~Hz}, 6 \mathrm{H}$, $\left.\mathrm{CH}_{3}\right), 1.41\left(\mathrm{t}, J=6.8 \mathrm{~Hz}, 3 \mathrm{H}, \mathrm{CH}_{3} \mathrm{CH}_{2} \mathrm{O}\right), 1.61-1.69(\mathrm{~m}, 4 \mathrm{H}$, piperidine $\mathrm{H}), 1.91(\mathrm{~d}, J=7.2 \mathrm{~Hz}, 1 \mathrm{H}$, cyclopropane $\mathrm{H}), 1.91$ (d, $J=7.2 \mathrm{~Hz}, 1 \mathrm{H}$, cyclopropane $\mathrm{H}$ ), 2.51-2.67 (m, 3H, piperidine $\mathrm{H}), 3.99-4.07$ (m, $\left.2 \mathrm{H}, \mathrm{CH}_{3} \mathrm{CH}_{2} \mathrm{O}\right)$, 6.85-6.87 (m, $\left.2 \mathrm{H}, \mathrm{Ph}\right)$, 7.42-7.49 (m, 2H, Ph); Ms m/z (relative intensity/\%): 369 ([M$\left.1]^{+}, 100\right), 256$ (95), 334 (33), 306 (29), 270 (36), 222 (36), 165 (37), 140 (67), 131 (46), 112 (39); Anal. calcd. for $\mathrm{C}_{19} \mathrm{H}_{25} \mathrm{NO}_{2} \mathrm{Cl}_{2}$ (\%): C 61.62, H 6.80, N 3.78; found: C 61.66, H 6.85, N 3.72 .

2,2-Dichloro-1-(4-ethoxyphenyl)cyclopropanyl 4-tertbutylpiperidin-1-yl ketone (4j). Yellow crystal; yield, 55.6 \%; m.p., 113-115 ${ }^{\circ} \mathrm{C} ;{ }^{1} \mathrm{H}$ NMR $\left(\mathrm{CDCl}_{3}\right) \delta: 0.80\left(\mathrm{~s}, 9 \mathrm{H}, \mathrm{CH}_{3}\right), 1.41$ $\left(\mathrm{t}, J=7.2 \mathrm{~Hz}, 3 \mathrm{H}, \mathrm{CH}_{3}\right), 1.60-1.73(\mathrm{~m}, 5 \mathrm{H}$, piperdine $\mathrm{H}), 1.99$ (d, $J=7.2 \mathrm{~Hz}, 1 \mathrm{H}$, cyclopropane H), 2.35 (d, $J=7.2 \mathrm{~Hz}, 1 \mathrm{H}$, cyclopropane $\mathrm{H}$ ), 2.93-3.01 (m, 4H, piperdine $\mathrm{H}$ ), 4.00-4.05 (m, $2 \mathrm{H}, \mathrm{CH}_{3} \mathrm{CH}_{2} \mathrm{O}$ ), 6.85-6.87 (m, 2H, Ph), 7.44-7.48 (m, $2 \mathrm{H}$, $\mathrm{Ph}) ; \mathrm{Ms} m / z$ (relative intensity/\%): 397([M-1] $\left.]^{+}, 100\right), 362$ (30), 334 (24), 298 (29), 222 (38), 168 (42), 131 (41), 102 (10); Anal. calcd. for $\mathrm{C}_{21} \mathrm{H}_{29} \mathrm{NO}_{2} \mathrm{Cl}_{2}(\%)$ : C 63.31, H 7.34, N 3.52, found: C 63.27, H 7.31, N 3.57.

Single crystal: The prism-shaped single crystal of the title compound was obtained by recrystallization from EtOH and $\mathrm{CH}_{2} \mathrm{Cl}_{2}$. The crystal with dimensions of $0.20 \mathrm{~mm} \times 0.16$ $\mathrm{mm} \times 0.12 \mathrm{~mm}$ was mounted on a Bruker SMART $1000 \mathrm{CCD}$ area-detector diffractometer ${ }^{5}$ with a graphite-monochromated $\operatorname{MoK}_{\alpha}$ radiation $(\lambda=0.71073 \AA)$ by using a Phi scan modes at 113 (2) $\mathrm{K}$ in the range of $2 \theta \leq 55.0^{\circ}$. The calculations were performed with SIR97 program and the empirical absorption corrections were applied to all intensity data. 


\section{RESULTS AND DISCUSSION}

The 1-(4-ethyoxyphenyl)-2,2-dichlorocyclopropane-1carboxylic acid, prepared according the reference. Cyclopropanecarbonyl chloride was prepared from the cyclopropane dicarboxylic acid and $\mathrm{SOCl}_{2}$, without isolation further reacted with substituted peridines at room temperature as shown in Scheme-I.

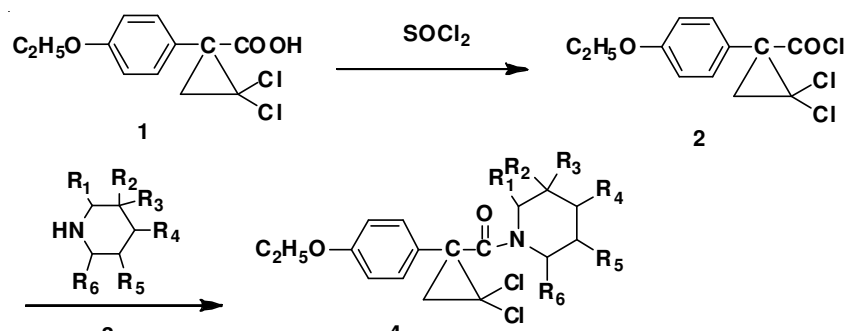

4a, $R_{1}=R_{2}^{3}=R_{3}=R_{4}=R_{5}=R_{6}=H ; 4 b, R_{1}=R_{2}=R_{3}=R_{5}=R_{6}=H, R_{4}=C H_{3} ; 4 c, R_{1}=R_{3}=R_{4}=R_{5}=R_{6}=H$, $\mathrm{R}_{2}=\mathrm{CH}_{3} 4 \mathrm{~d}, \mathrm{R}_{2}=\mathrm{R}_{3}=\mathrm{R}_{4}=\mathrm{R}_{5}=\mathrm{R}_{6}=\mathrm{H}, \mathrm{R}_{1}=\mathrm{CH}_{2} \mathrm{CH}_{3} ; 4 \mathrm{e}, \mathrm{R}_{1}=\mathrm{R}_{2}=\mathrm{R}_{3}=\mathrm{R}_{5}=\mathrm{R}_{6}=\mathrm{H}, \mathrm{R}_{4}=\mathrm{CH}_{2} \mathrm{CH}_{3}$;

4f, $R_{2}=R_{3}=R_{4}=R_{5}=H, R_{1}=R_{6}=C_{3} ; 4$ g, $R_{1}=R_{4}=R_{5}=R_{6}=H, R_{2}=R_{3}=C_{3} ; 4 h, R_{3}=R_{4}=R_{5}=R_{6}=H$, $R_{1}=R_{2}=C_{3} 4 i, R_{1}=R_{3}=R_{4}=R_{6}=H, R_{2}=R_{5}=C_{3} ; 4 j, R_{1}=R_{2}=R_{3}=R_{5}=R_{6}=H, R_{4}=C\left(C_{3}\right)_{3}$

Scheme-I: Synthetic route of title compounds

Crystal structures of 4a: The crystal symmetry is monoclinic, space group $\mathrm{P} 2{ }_{1} / \mathrm{n}$, with $\mathrm{a}=0.60819$ (19) $\mathrm{nm}, \mathrm{b}=1.5592$ (6) $\mathrm{nm}, \mathrm{c}=1.8000$ (7) nm, $\alpha=90^{\circ}, \beta=91.087(14)^{\circ}, \gamma=90^{\circ}$, $\mathrm{V}=1.7066(10) \mathrm{nm}^{3}, \mathrm{Z}=4, \mathrm{Dx}=1.332 \mathrm{Mg} / \mathrm{m}^{3}, \mu=0.39 \mathrm{~mm}^{-1}$, $\mathrm{R}=0.035, \mathrm{wR}=0.081$. The single crystal of the title compounds was obtained and its structure is shown in Fig. 1. The torsion angles of C2-C1-C4-O1 and C3-C1-C4-O1 are 65.82 (17) $^{\circ}$ and $133.21(14)^{\circ}$ respectively. From the molecular structure, it can be seen that the piperidine ring is in the e-bond positions of chair conformation in the six membered ring.

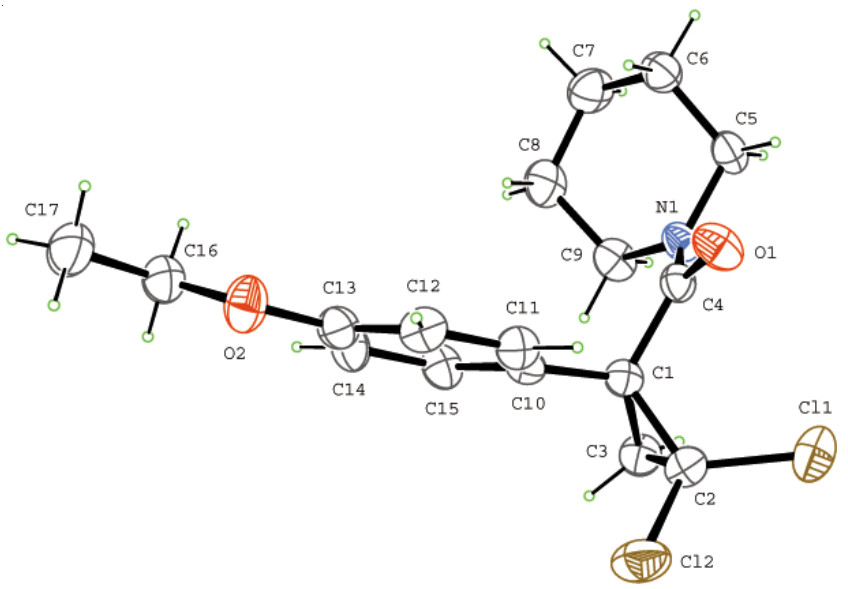

Fig. 1. Molecular structure of $\mathbf{4 a}$

Crystal structures of $4 \mathrm{~h}$ : The crystal symmetry is monoclinic, space group $\mathrm{P} 2{ }_{1} / \mathrm{n}$, with $\mathrm{a}=1.1662$ (5) $\mathrm{nm}, \mathrm{b}=1.6242$ (8) nm, c $=1.1398$ (5) nm, $\alpha=90^{\circ}, \beta=115.078(14)^{\circ}, \gamma=90^{\circ}$, $\mathrm{V}=1.9553(10) \mathrm{nm}^{3}, \mathrm{Z}=4, \mathrm{Dx}=1.258 \mathrm{Mg} / \mathrm{m}^{3}, \mu=0.34 \mathrm{~mm}^{-1}, \mathrm{R}$ $=0.034, \mathrm{wR}=0.085$. The single crystal of the title compounds was obtained and its structure is shown in Fig. 2. The torsion angles of C2-C1-C4-O1 and C3-C1-C4-O1 are-125.0(2) and $-57.2(2)^{\circ}$ respectively. From the molecular structure, it can be seen that the piperidine ring is in the e-bond positions of chair conformation in the six membered ring. The dihedral angle of cyclpopropane and phenyl is $55.68(17)^{\circ}$. In addition, X-ray analysis reveals that there exist three intermolecular hydrogen bonds in the crystal. The intermolecular hydrogen bond lengths of $\mathrm{C} 11-\mathrm{H} \cdots \mathrm{O} 1$ is $3.322(2) \AA$.

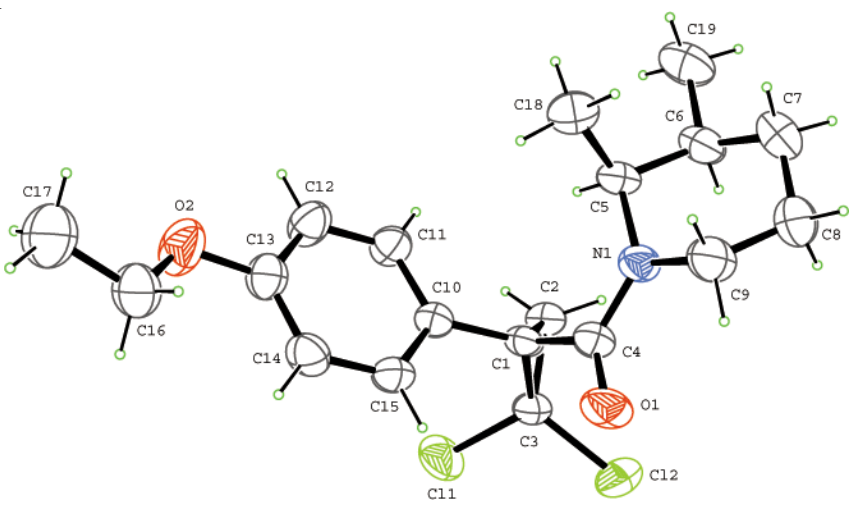

Fig. 2. Molecular structure of $4 \mathrm{~h}$

Fungicidal activity: The in vivo fungicidal results of all of the compounds against Rhizoctonia solani, Pseudoperonospora cubensis, Sphaerotheca fuliginea and Botrytis cinerea were listed in Table-1. As shown in Table-1, all these compounds did not display obvious fungicidal activities against Rhizoctonia solani, Pseudo- peronospora cubensis, Sphaerotheca fuliginea. Among them, these compounds displayed the highest fungicidal activity against Botrytis cinerea. Compounds $\mathbf{4 b}$, $\mathbf{4 c}, \mathbf{4 i}$ have fair to moderate fungicidal activity against Botrytis cinerea. at the concentration of $200 \mu \mathrm{g} \mathrm{mL} \mathrm{L}^{-1}$.

\begin{tabular}{ccccc}
\multicolumn{5}{c}{ TABLE-1 } \\
FUNGICIDAL ACTIVITIES OF 4 (INHIBITION/\%) \\
\hline Compd. & $\begin{array}{c}\text { Sphaerotheca } \\
\text { fuliginea }\end{array}$ & $\begin{array}{c}\text { Pseudo- } \\
\text { peronospora } \\
\text { cubensis }\end{array}$ & $\begin{array}{c}\text { Botrytis } \\
\text { cinerea }\end{array}$ & $\begin{array}{c}\text { Rhizoctonia } \\
\text { solani }\end{array}$ \\
\hline $\mathbf{4 a}$ & 0.00 & 0.00 & 17.25 & 0 \\
$\mathbf{4 b}$ & 3.11 & 6.53 & 45.36 & 0 \\
$\mathbf{4 c}$ & 0.00 & 7.58 & 59.85 & 0 \\
$\mathbf{4 d}$ & 6.74 & 0.00 & 28.61 & 0 \\
$\mathbf{4 e}$ & 8.79 & 3.21 & 30.80 & 0 \\
$\mathbf{4 f}$ & 1.63 & 6.39 & 21.53 & 0 \\
$\mathbf{4 g}$ & 0 & 0 & 0.00 & 0 \\
$\mathbf{4 h}$ & 0 & 0 & 0.00 & 0 \\
$\mathbf{4 i}$ & 0 & 0 & 43.66 & 0 \\
$\mathbf{4 j}$ & 0 & 0 & 34.86 & 0 \\
\hline
\end{tabular}

\section{ACKNOWLEDGEMENTS}

This work was funded by the Program of Education Department of Zhejiang Province, China (Y200803060).

\section{REFERENCES}

1. a) X.H. Liu, C.X. Tan, J.Q. Weng and H.J. Liu, Acta Cryst., E68, o493(2012); b) X.H. Liu, P.Q. Chen, B.L. Wang, Y.H. Li and Z.M. Li, Bioorg. Med. Chem. Lett., 17, 3784 (2007); c) X.H. Liu, P.Q. Chen, F.Q. He, Y.H. Li, S.H. Wang and Z.M. Li, Struct. Chem., 5, 563 (2007); d) X.H. Liu, C.Y. Zhang, W.C. Guo, Y.H. Li, P.Q. Chen, T. Wang, W.L. Dong, B.L. Wang, H.W. Sun and Z.M. Li, J. Enzym. Inhib. Med. Chem., 24, 545 (2009); e) X.H. Liu, Y.X. Shi, Y. Ma, G.R. He, W.L. Dong, C.Y. Zhang, B.L. Wang, S.H. Wang, B.J. Li and Z.M. Li, Chem. Biol. Drug Des., 73, 320 (2009); f) X.H. Liu, Y.X. Shi, Y. Ma, C.Y. Zhang, W.L. Dong, P. Li, B.L. Wang, B.J. Li and Z.M. Li, Eur. J. Med. Chem., 
44, 2782 (2009); g) X.H. Liu, J.Q. Weng, C.X. Tan, L. Pan, B.L. Wang and Z.M. Li, Asian J. Chem., 23, 4031 (2011); h) H.J. Liu, J.Q. Weng, C.X. Tan and X.H. Liu, Acta Cryst., E67, o1940 (2011).

2. a) X.H. Liu, L. Pan, J.Q. Weng, C.X. Tan, Y.H. Li, B.L. Wang and Z.M Li, Mol. Divers., 16,251(2012); b) C.X. Tan, Y.X. Shi, J.Q. Weng, X.H. Liu, B.J. Li and W.G. Zhao, Lett. Drug Des. Discov., 9, 431 (2012); c) X.H. Liu, L. Pan, C.X. Tan, J.Q. Weng, B.L. Wang and Z.M. Li, Pestic. Biochem. Physiol., 101, 143 (2011); d) C.X. Tan, Y.X. Shi, J.Q. Weng, X.H. Liu, B.J. Li and W.G. Zhao, J. Heterocycl. Chem., DOI:10.1002/ jhet.1656.

3. K. Matsuda, K. Iharada, K.H. Suzuki, M. Yamashita, H. Okimoto, K. Nishimura, K.T. Ueno and K. Komai, J. Pestic. Sci., 20, 487 (1995).

4. a) X.H. Liu, C.X. Tan and J.Q. Weng, Phosphorus Sulfur Silicon Rel. Elem., 186, 552 (2011); b) X.H. Liu, C.X. Tan and J.Q. Weng, Phos- phorus Sulfur Silicon Rel. Elem., 186, 558 (2011); c) X.H. Liu, L. Pan, Y. Ma, J.Q. Weng, C.X. Tan, Y.H. Li, Y.X. Shi, B.J. Li, Z.M. Li and Y.G. Zhang, Chem. Biol. Drug Des., 78, 689 (2011); d) P.Q. Chen, C.X. Tan, J.Q. Weng and X.H. Liu, Asian J. Chem., 24, 2808 (2012); e) J.Q. Weng, C.X. Tan, L. Wang and X.H. Liu, J. Chem. Soc. Pak., 34, 1248(2012); f) Y.L. Xue, Y.G. Zhang and X.H. Liu, Asian J. Chem., 24, 3016 (2012); g) Y.L. Xue, Y.G. Zhang and X.H. Liu, Asian J. Chem., 24, 1571 (2012); h) X.F. Liu and X.H. Liu, Acta Cryst., E67, o202 (2011).

5. Bruker, Smart-NT. Version 5.05 Bruker AXS inc, Madsion, Wisconsin, USA (1998). 